\title{
Intermittent fasting induces rapid hepatocyte proliferation
}

\author{
Abby Sarkar ${ }^{1 *}$, Yinhua Jin ${ }^{1}$, Teni Anbarchian ${ }^{1}$, Yan Yang ${ }^{2}$, Huy Nguyen ${ }^{3}$, Eric Rulifson ${ }^{1}$, Matt \\ Fish $^{1}$, Catriona Y. Logan ${ }^{1}$, Avi Kaye ${ }^{1}$, Peng Wu ${ }^{1,4}$, Roel Nusse ${ }^{1 *}$ \\ ${ }^{1}$ Howard Hughes Medical Institute, Department of Developmental Biology, Institute for Stem \\ Cell Biology and Regenerative Medicine, Stanford University School of Medicine, Stanford, CA \\ 94305, USA. \\ ${ }^{2}$ Stanford Center for Genomics \& Personalized Medicine, Stanford University School of \\ Medicine, Stanford, CA 94305, USA. \\ ${ }^{3}$ Department of Neurology and Neurological Sciences, Stanford University School of Medicine, \\ Stanford, CA 94305, USA, \\ ${ }^{4}$ Department of Pediatrics, Stanford University School of Medicine, Stanford, CA 94305, USA. \\ *Correspondence to: rnusse@stanford.edu; ajsarkar@stanford.edu
}

\begin{abstract}
Nutrient availability fluctuates in most natural populations, forcing organisms to undergo periods of fasting and re-feeding. It is unknown how dietary change influences cell proliferation in the liver. Here, we show that intermittent fasting (IF) promotes rapid hepatocyte proliferation. Mechanistically, IF elicits intestinal production of endocrine FGF15, which activates the $\beta$ KLOTHO receptor on hepatocytes to induce proliferation. Furthermore, IF-induced proliferation is locally controlled in hepatocytes by WNT signaling and the WNT target gene Tbx3. IF induces hepatocyte proliferation to re-establish a constant liver-to-body-mass ratio during periods of fasting and re-feeding and maintain the hepatostat. This study provides the first example of dietary influence on adult hepatocyte proliferation and identifies both systemic FGF and local WNT as regulators of this process.
\end{abstract}

\section{Introduction}

Periods of fasting and re-feeding induce profound tissue remodeling and regeneration in several tissues including the intestine (O'Brien et al. 2011; Yilmaz et al. 2012), the muscle (Cerletti et al. 2012) and blood (Brandhorst et al. 2015; Chen et al. 2003; Ertl et al. 2008). These tissue changes are thought to be mediated through diet-induced growth factor signaling, including both local, paracrine and systemic, endocrine signals, that influence cell biology and function (Mihaylova et al. 2014). The impact of fasting and re-feeding on liver tissue homeostasis is unknown.

The many metabolic functions of the liver are executed by hepatocytes. Organized into hexagonal lobular units, hepatocytes are stacked in between a central vein and a portal triad, that consists of a portal vein, hepatic artery, and bile duct. The directional flow of oxygenated blood from the portal to central axis creates a gradient of cytokines, nutrients and growth factors throughout the liver lobule that influences hepatocyte transcriptome and function (Halpern et al. 2017). Thus, pericentral hepatocytes, present near the central vein, receive different growth factor signals compared to the midlobular and periportal hepatocytes that occupy the rest of the 
liver lobule. Hepatocyte turnover along the liver lobule has been well characterized during ad libitum (AL) feeding (Chen et al. 2020; He et al. 2021; Lin et al. 2018; Wang et al. 2015; Wei et al. 2021), when animals are given constant access to food. In these studies hepatocytes have an appreciable rate of turnover and division (He et al. 2021; Wei et al. 2021), but the proliferation rates compared to other tissues are low (Michalopoulos 2021). No study so far has looked at hepatocyte turnover during periods of fasting and re-feeding, arguably a dietary state that more closely mimics nutrient availability and intake of natural populations.

\section{Results}

\section{Intermittent fasting induces rapid proliferation of pericentral hepatocytes}

To determine the impact of IF on hepatocyte proliferation, we employed a system of random cell lineage tracing to capture a representative sample of all cells and trace their clonal expansion in the liver under an AL or IF feeding regimen. We used an inducible, R26-CreERT2 (Ventura et al. 2007) allele to permanently label cells with one of the four fluorophores in the R26-Confetti allele (Snippert et al. 2010). Following two weeks of tamoxifen clearance from the liver (fig. S1A), livers were collected from R26-CreERT2/Confetti mice right after clearance (T0) or 1-3 weeks after AL or IF feeding regimens (Fig. 1A). Beginning at T0, greater than $95 \%$ of labeled cells co-expressed one of the fluorophores and the hepatocyte specific transcription factor HNF4A (Fig. 1B), demonstrating labeling of mostly single hepatocytes distributed throughout the liver lobule (Fig. 1C). 1-3 weeks after AL or IF feeding regimens, distinct clones of 2 or more cells expressing the same label grew both in AL and IF-treated animals. Markedly, there was an increased number of cells per clone in IF animals compared to AL animals (Fig. 1, B and C). Furthermore, at 3 weeks multicellular clone occurrence in IF-treated animals was significantly increased in the pericentral region of the liver lobule (Fig. 1D). The frequency of clone occurrence in each region of the liver lobule did not appreciably change between time points or feeding regimens (Fig. S1B). This suggested ectopic labeling after tamoxifen clearance was rare and that hepatocyte streaming, proliferation on one end of the lobule and death on the other end, did not occur. In support of the of the rapid, pericentrally-localized clonal expansion observed in R26-CreT2/Confetti livers, the cell cycle marker Ki67 was detected in pericentral hepatocytes in 1 week IF-treated mice during re-feeding (Fig. S1C).

To identify transcriptional changes that occur as a consequence of IF-induced hepatocyte proliferation, we performed single cell-RNA sequencing and in situ hybridization on livers from 1 week IF-treated and AL-treated animals. Livers were collected during a matched, neutral feeding and circadian state. Sequenced hepatocytes were classified into pericentral, midlobular and periportal hepatocytes (Fig. S1D), using well characterized marker genes (Halpern et al. 2017). The proportion of hepatocytes enriched for pericentral transcripts in IF-treated animals was $19.52 \pm 4.9 \%$, more than twice as much as the proportion observed in AL-treated animals, $8.97 \pm 1.2 \%$ (Fig. S1E). The increased proportion of pericentral hepatocytes in IF-treated animals was confirmed by in situ hybridization (Fig. S1F). 


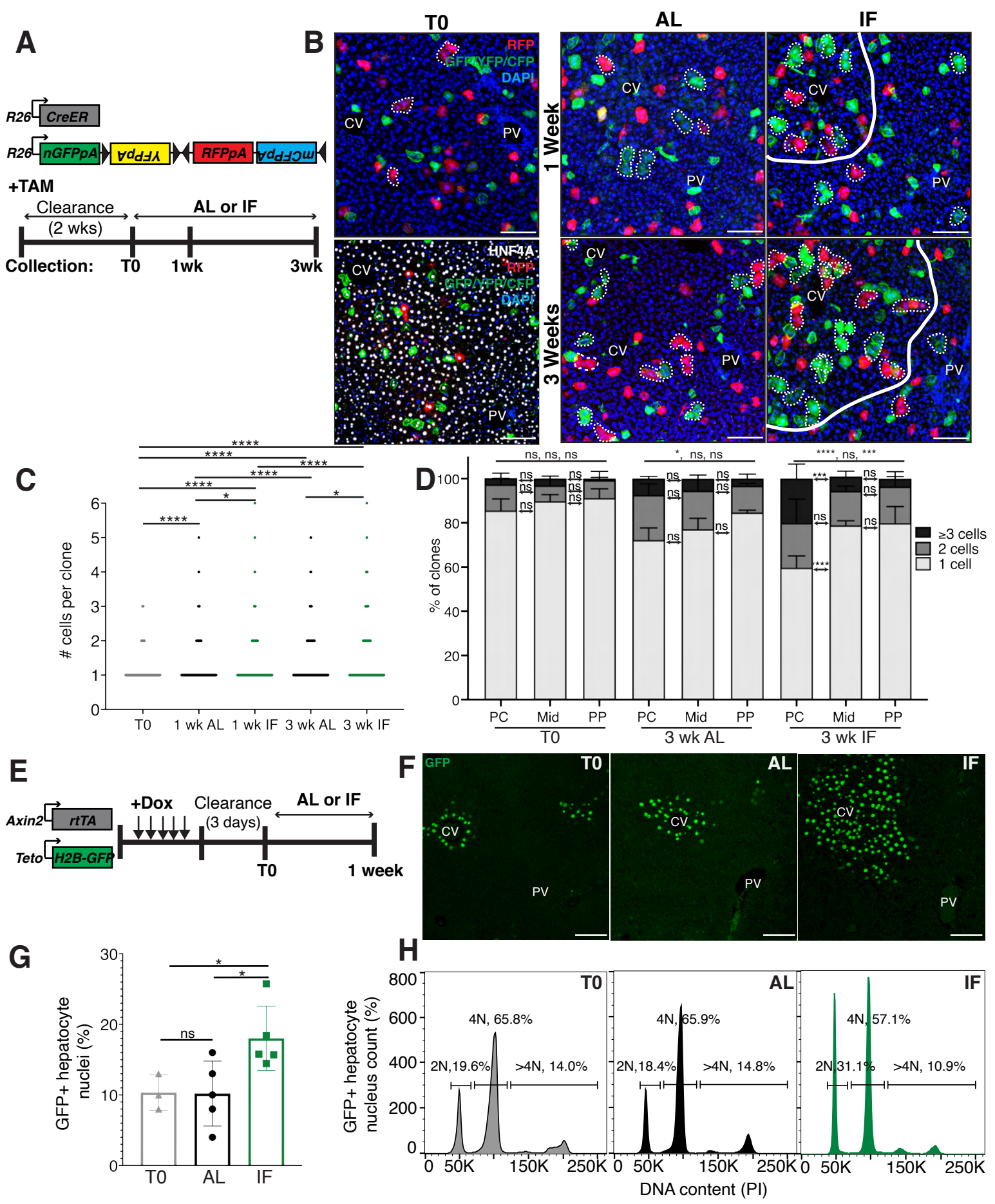

Figure 1. Intermittent fasting induces rapid proliferation of hepatocytes in adult liver. (A) Schematic of unbiased system to trace cell proliferation during ad libitum feeding (AL) and intermittent fasting (IF). R26-CreER mice were crossed to R26-Confetti mice enabling permanent cell labeling and lineage tracing by 4 fluorescent reporters after tamoxifen administration. (B) At T0, mostly single, HNF4A+ hepatocytes were labeled throughout the lobule. At 1 week and 3 weeks, multicellular hepatocytes clones (dotted circles) grew in AL and IF livers, with increased pericentral growth in IF. (C) Number of hepatocytes per 3D clone at each collection in A. Mann-Whitney test. 439-602 clones analyzed at T0, 430-1085 clones at $1 \mathrm{wk}$ AL, 625-904 clones at $1 \mathrm{wk}$ IF, 523-615 clones at 3 wk AL, and 442-615 at 3 wk IF. N=3. (D) Percentage of 3D clones consisting of different cell sizes, from C, in 
different liver lobule locations. PC, pericentral; Mid, midlobular; PP, periportal. Differences between 1-, 2- and >3 cell clones in periportal and pericentral zones are indicated by $\mathrm{P}$ values above bars. 2 -way ANOVA. N=3. (E) Doxycycline inducible Axin2-rtTA; Teto-H2BGFP system to label all pericentral hepatocytes and trace cell proliferation. Mice were pulsed with doxycycline (Dox) for 7 days, cleared of dox for 3 days and ad libitum fed or intermittently fasted for 6 days. (F) GFP immunofluorescent images showing increased hepatocyte expansion in AL and IF compared to T0. (G) Percentage of GFP+ hepatocyte nuclei in AL, IF livers from A. One-way ANOVA. N=3 (T0), 5 (AL), 5 (IF). (H) Nuclear ploidy distribution of GFP+ hepatocytes in T0, AL and IF livers. $* * * * \mathrm{P}<0.0001$; $* * * \mathrm{P}<0.001 ; * \mathrm{P}<0.05$; ns., not significant. Error bars indicate standard deviation. Scale bar, $100 \mu \mathrm{m}$. wk, weeks.

To study pericentral hepatocyte proliferation kinetics during ad libitum feeding and intermittent fasting, we utilized an inducible system to mark and trace pericentral hepatocytes. In Axin2-rtTA; TetO-H2B-GFP transgenic mice (Tumbar et al. 2004; Yu et al. 2007), a modified promoter of the Wnt transcriptional target gene, Axin2, is used to control expression of a stable histone 2B-GFP fusion protein with doxycycline administration, thus marking WNT-responsive, pericentral hepatocytes and their progeny (8). Importantly, in these mice the ectopic Axin2-rtTA expression cassette leaves the endogenous Axin2 locus unchanged. Axin2rtTA; TetO-H2BGFP animals were given doxycycline(dox) for seven days, then cleared of dox for 3 days, and analyzed after dox clearance (T0) or after an additional 6 days of AL or IF feeding regimen (Fig. 1E and S1G). We observed a 74\% increase in GFP-labeled hepatocyte nuclei in IF-treated animals compared T0 animals (Fig. 1F, G). No significant change was observed between AL and T0 animals. Hepatocyte division is coordinated with changes in nuclear ploidy in the liver (Matsumoto et al. 2020; Miyaoka et al. 2012). To test if IF-treatment changed nuclear ploidy of WNT-responsive cells and progeny, we FACS sorted GFP-labeled nuclei of hepatocytes from Axin2rtTA; TetO$H 2 B G F P$ mice at T0 and 1 week after AL or IF treatment and evaluated ploidy status with propidium iodide staining (Fig. $1 \mathrm{H})$. There was a $58 \%$ increase in the percentage of diploid hepatocyte nuclei in IF-treated animals relative to T0 animals, and overall nuclear ploidy decreased in IF-treated animals (Fig. 1H). As a control, ploidy in AL animals was similar to T0 (Fig. 1H). Together these data demonstrate the enhanced proliferation of pericentral hepatocytes with an IF feeding regimen.

\section{Endocrine FGF15- $\beta$-KLOTHO signaling is required for hepatocyte proliferation during intermittent fasting}

To understand the underlying mechanism for increased pericentral hepatocyte proliferation with IF feeding, we first examined known signaling pathways involved in nutrient sensing. Endocrine FGF signaling is critical in mediating an organism's physiological response to fasting and refeeding (Potthoff et al. 2012). Upon re-feeding, FGF15, produced in intestinal enterocytes, travels through the blood stream and binds to its co-receptor, $\beta$-KLOTHO, on hepatocytes (Inagaki et al. 2005). Endocrine FGF signaling has also been shown to play important roles in regulating hepatocyte metabolism (Inagaki et al. 2005; Potthoff et al. 2011) and regeneration (Uriarte et al. 2013). Given that endocrine FGF15 is an early regulator of the hepatocyte response to food intake, we asked whether loss of endocrine FGF signaling in hepatocytes would prevent IF-induced proliferation. To test this, we genetically depleted hepatocytes of the endocrine FGF receptor, $\beta$-Klotho $(K l b)$ and traced expansion of Axin2+ GFP-labeled cells during IF-treatment (Fig. 2A). Loss of $K l b$ led to a 53\% reduction in GFP-labeled pericentral hepatocyte nuclei compared to control animals after 1 week of an IF feeding regimen (Fig. 2A and B). Importantly, loss of $K l b$ did not impact WNT target gene expression (Fig. 2C), a critical regulator of hepatocyte zonation and function in the liver (Perugorria et al. 2019; Wang et al. 
2015). Furthermore, loss of $K l b$ did not significantly change GFP-labeled nuclei ploidy (Fig. 2D). These results suggest that FGF15 acts as an inducer of hepatocyte division.

To directly test the impact of FGF15 on hepatocyte division, we ectopically expressed Fgf15 in the liver under ad libitum feeding (Fig. 2E and F). Indeed, treatment of animals with AAV-TTRFGF15 led to a $102 \%$ percent increase in Axin2+ GFP-labeled nuclei compared to control animals, (Fig. 2G). In summary, these data emphasize the functional requirement for endocrine FGF15- $\beta$-KLOTHO signaling to promote IF-induced pericentral hepatocyte proliferation.
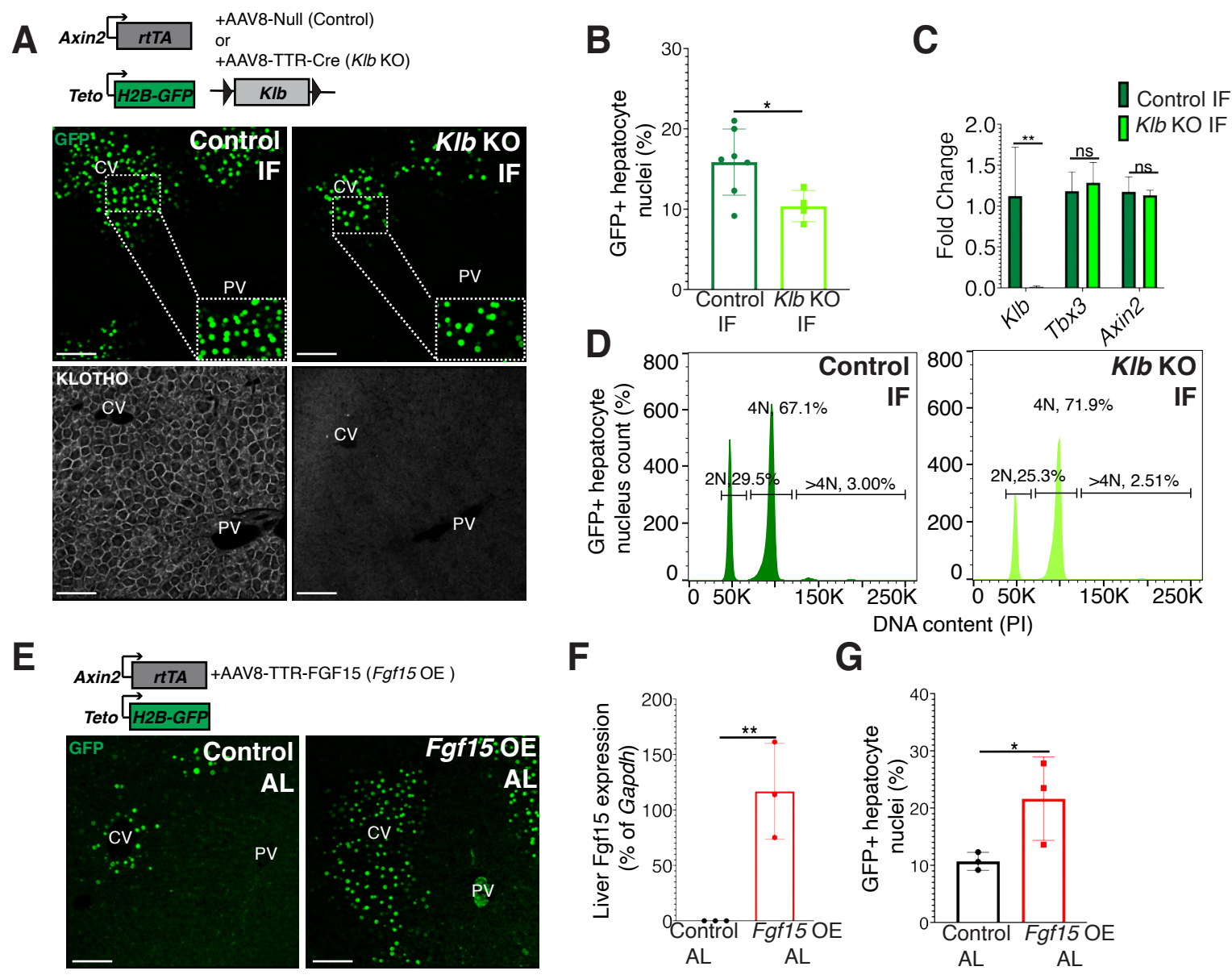

$\mathbf{F}$
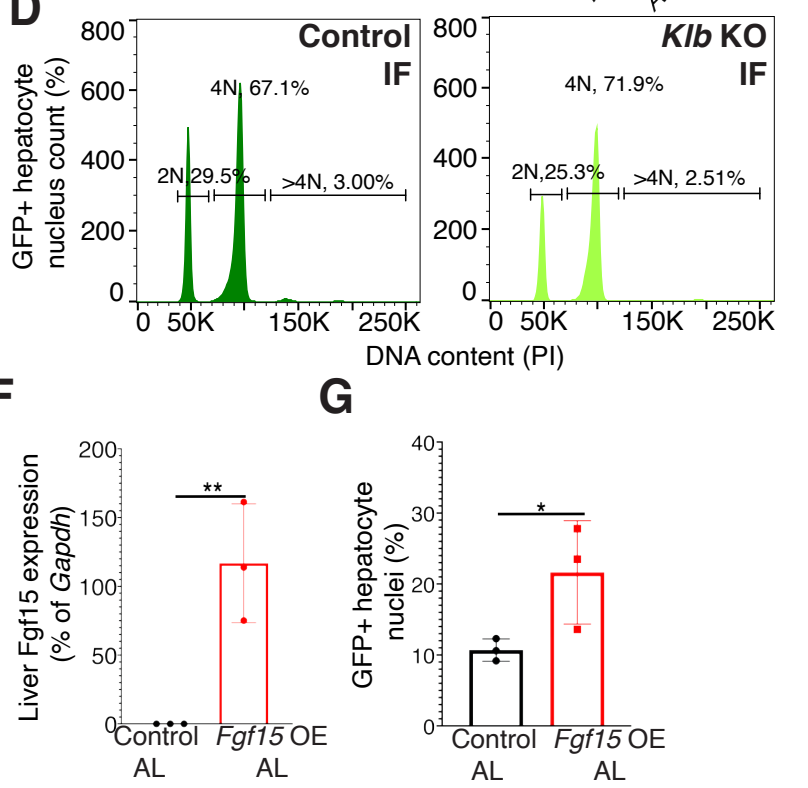

Figure 2. Endocrine FGF15-8-KLOTHO signaling is required for hepatocyte expansion during intermittent fasting. (A) Schematic of method to deplete hepatocytes of Klb. Axin2-rtTA; Teto-H2BGFP; Klbflox/flox mice were injected with AAV-TTR-Null (Control) or AAV-TTR-Cre (Klb KO). GFP and KLOTHO immunofluorescent images showing decrease in hepatocyte expansion and loss of KLOTHO in Klb KO compared to Control livers. (B) Percentage of GFP+ hepatocyte nuclei in Klb KO and Control livers. Unpaired t-test. N=7 (Control), 4 (Klb KO). (C) Quantitative real-time PCR analysis of $K l b$, Tbx3 and Axin2. Unpaired t-test, N=3. (D) Ploidy distribution of GFP+ hepatocyte nuclei in Control IF and Klb KO IF livers. (E) Schematic for Fgf15 overexpression. Axin2-rtTA; Teto-H2BGFP mice were injected with AAV-TTR-FGF15 (Fgf15 OE). GFP immunofluorescent images from AL and Fgf15 OE AL-treated livers. (F) Quantitative real-time PCR analysis of Fgf15. Unpaired t-test. N=3. (G) Percentage of GFP+ hepatocyte nuclei in $\mathrm{AL}$ and $\mathrm{Fgfl} 15 \mathrm{OE}$ livers. Unpaired t-test, N=3. ${ }^{* *} \mathrm{P}<0.01,{ }^{*} \mathrm{P}<0.05$, ns., not significant. Error bars indicate standard deviation. Scale bar, $100 \mu \mathrm{m}$. 
Intermittent fasting promotes enhanced pericentral hepatocyte proliferation through WNT signaling and the WNT target gene Tbx3

Next, we sought to understand why pericentral hepatocytes preferentially divided in response to IF, as FGF15 is a hormone and would be theoretically accessible to all hepatocytes. One hypothesis is that IF-induced hepatocyte proliferation additionally requires a second signal, which is concentrated near pericentral hepatocytes. Pericentral hepatocytes receive paracrine WNT signals from endothelial cells of the central vein, which is required to establish and maintain pericentral hepatocyte zonation and function in the liver (Perugorria et al. 2019; Wang et al. 2015). We tested whether ectopic and constitutive activation of the WNT pathway in midlobular and periportal hepatocytes, which typically do not receive WNT signaling, would promote hepatocyte proliferation under an IF feeding regimen. We injected mice ubiquitously expressing Cas9 with an AAV8-U6-sgApc construct expressing a guide RNA against the WNT repressor gene Apc (Fig. 3A). Midlobular and periportal hepatocytes that had undergone CRISPR-Cas 9 gene editing of $A p c$, were identified by the WNT-target glutamine synthetase (GS). Remarkably, these WNT activated (GS+) cells remained mostly as single cells in AL treatment, however they clonally expanded under IF treatment (Fig. 3A and B).

WNT signaling induces expression of the transcriptional repressor Tbx3 in adult hepatocytes (fig. S2A). Previous studies have demonstrated that $T b x 3$ regulates division of hepatoblasts during embryonic liver development by repressing cell cycle inhibitors (Suzuki et al. 2008). In an accompanying manuscript, we show that $T b x 3$ is required for hepatocyte cell division during postnatal development (Jin et al. 2021). To determine if Tbx3 plays a role in IF-induced hepatocyte proliferation, we genetically depleted $T b x 3$ in hepatocytes and traced expansion of Axin2+ GFP-labeled cells after IF-treatment (Fig 3C). Loss of Tbx3 led to a 51\% reduction in expansion of GFP-labeled cells after IF (Fig. 3D). Furthermore, loss of Tbx3 increased nuclear ploidy in GFP-labeled cells, suggesting that pericentral hepatocytes underwent endoreplication rather than division (Figure $3 \mathrm{E}$ and $\mathrm{F}$ ). These findings suggest that WNT and WNT-induced transcription factor TBX3 endow pericentral hepatocytes with capacity to divide and maintain normal liver homeostasis during IF treatment. 

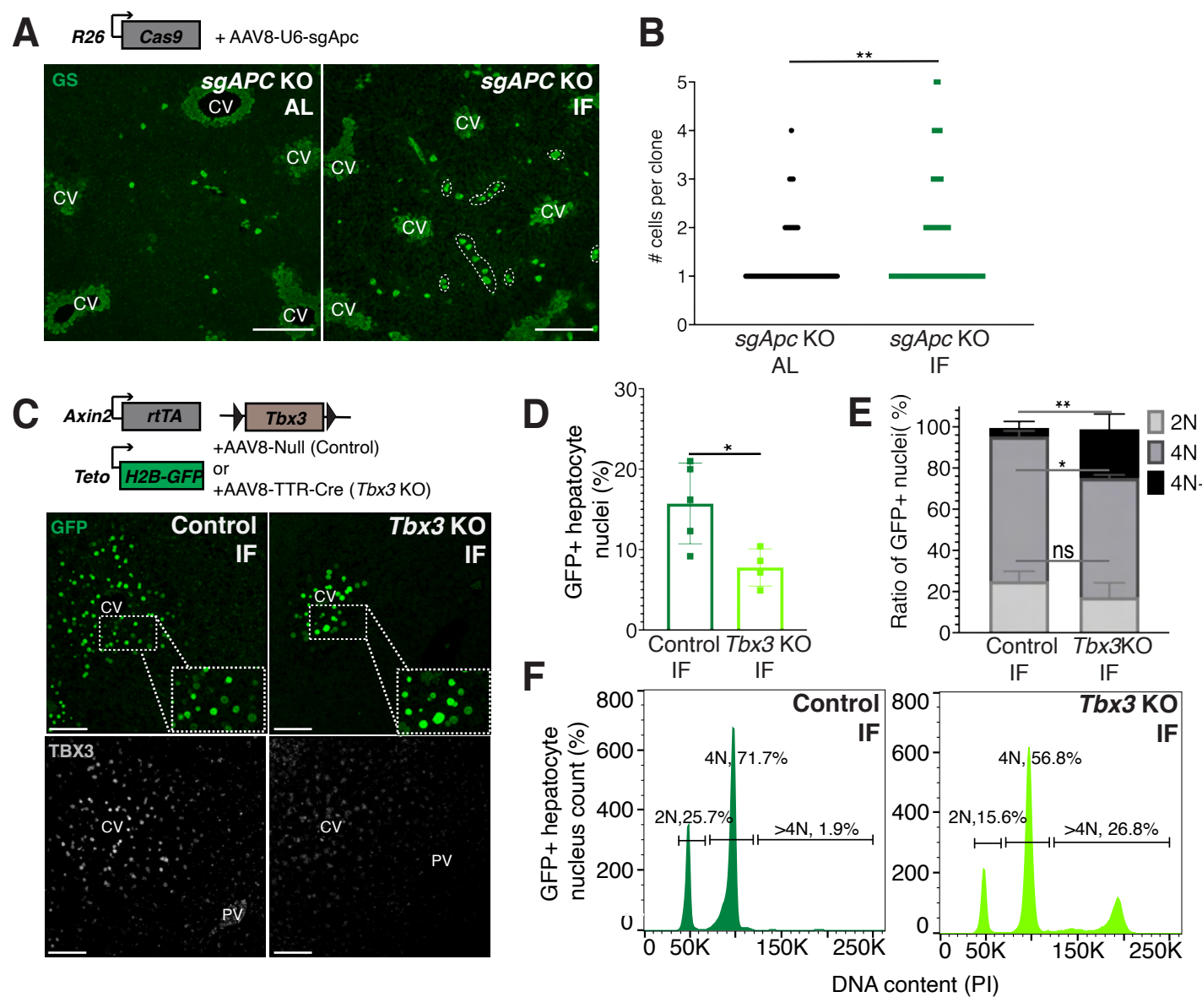

Figure 3. Paracrine WNT acts as a permissive signal for hepatocyte proliferation during intermittent fasting. (A) A method to constitutively activate WNT signaling in midlobular, periportal cells. AAV8-U6-sgAPC was injected into the tail vein of R26-Cas 9 mice. Animals were IF-treated for 1 week before analysis. GS immunofluorescent images for detection of Apc mutant clones in AL and IF livers . (B) Number of Apc mutant hepatocytes per 3D clone in AL and IF livers. Mann-Whitney test. N=3. (C) Schematic of method to deplete hepatocytes of the WNT target, TBX3. Axin2-rtTA; Teto-H2BGFP; Tbx3flox/flox mice were intraperitoneally injected with AAV8-Null (Control) or AAV8-TTR-Cre (Tbx3 KO). GFP and TBX3 immunofluorescent images to show IF-induced proliferation and Tbx3 depletion, respectively, in Control and Tbx3 KO livers. (D) Percentage of GFP+ hepatocyte nuclei in Control IF and Tbx3 KO IF livers. Unpaired t-test, $\mathrm{N}=7$ (Control IF), N=4(Tbx3 KO IF). (E and F) Nuclear ploidy distribution of GFP+ hepatocytes in Control IF and Tbx3 KO IF livers. Two-way ANOVA. $* * \mathrm{P}<0.01 .{ }^{*} \mathrm{P}<0.05$, ns., not significant. Error bars indicate standard deviation. Scale bar, $100 \mu \mathrm{m}$.

\section{Intermittent fasting disrupts the hepatostat}

During partial hepatectomy where two thirds of the liver is removed or during liver transplantation from a smaller organism to a larger one, loss of liver cell mass disrupts the hepatostat, the liver-to-body-weight ratio required to maintain homeostasis (Michalopoulos and Bhushan 2021). Re-establishment of the hepatostat through hepatocyte regeneration is critical to prevent development of liver disease (Michalopoulos and Bhushan 2021). We asked whether the hepatostat was disrupted during intermittent fasting. For early timeframes of IF (2-6 days), liverto-body weight ratio significantly decreased during fasting and increased during re-feeding states compared to AL-treated livers (Fig. 4A). However, after 3 weeks of IF-treatment, this ratio stabilized and was not significantly different between fasting, re-feeding or AL states. Histological analysis during early timeframes of IF-treatment revealed an initial decrease in 
hepatocyte size during fasting and an increase in hepatocyte size during re-feeding periods compared to AL treatment (Fig. 4B and C). Additionally, a distinct change in hepatocyte morphology was observed between IF-treated and AL-treated livers, where IF-treated hepatocytes appeared edematous with alterations in mitochondrial and ribosomal distribution (Fig. 4D and E). Hepatocyte edema in IF-treated livers did not appear to stem from an increase in glycogen or lipids content during re-feeding periods of IF (Fig. 4E). These findings suggest that intermittent fasting disrupts the hepatostat and that hepatocytes undergo both proliferation and hypertrophy, by swelling, to re-establish a constant liver-to-body weight ratio during fasting and re-feeding periods. To test the importance of hepatocyte proliferation on maintaining the liver-tobody weight ratio during IF, we compared this ratio between livers depleted of $K l b$ or $T b x 3$ in ad libitum fed or intermittently fasted animals (Fig. 4F). In AL-treated animals, loss of Klb did not significantly alter the liver-to-body weight ratio compared to control livers. In contrast, in IFtreated animals, loss of $K l b$ lead to a significant decrease in the liver-to-body ratio. Livers depleted of $T b x 3$ were able to maintain a ratio similar to control livers during AL and IFtreatment. These findings highlight the importance of hepatocyte proliferation during IF, and the ability of $T b x 3 \mathrm{KO}$ hepatocytes to undergo enhanced endoreplication and hypertrophy as a mechanism to maintain liver size in the absence of full hepatocyte division.

\section{Discussion}

Our data demonstrate that the liver is exquisitely tuned to changes in nutrient status and displays robust homeostatic mechanisms to ensure a constant liver to body weight ratio. In response to intermittent fasting, hepatocytes integrate nutrient sensing responses, mediated via intestinal FGF15 and knowledge of cellular position, mediated by local, pericentral WNT signals to proliferate. Pericentral hepatocyte proliferation ensures replacement of lost cellular mass through increases in overall cell numbers. Together with the work in an accompanying paper (Jin et al. 2021) we propose our current working model in which paracrine WNT and endocrine FGF pathways work together to push hepatocytes through different phases of the cell cycle. Specifically, the general role of FGF in cell proliferation observed in our studies suggest that this signal initiates S-phase, whereas WNT through TBX3 permits progression through Mphase by repressing cell division inhibitors. It has been demonstrated in other contexts as well that both FGF and WNT signaling are conjointly required for tissue growth (McGrew et al. 1997; ten Berge et al. 2008).

It will be of interest to know how the hepatostat is maintained during other nutrient conditions such as ketogenic, calorie restricted, or high-fat diets. Many tissues in the body are known to be slowly proliferating, but this conclusion has mainly stemmed from AL-fed mouse studies, which may not mimic the intermittent fasting that wild animals are exposed to. These studies demonstrate that there is perhaps more proliferative capacity than previously appreciated in tissues that are currently thought to exhibit a slow turnover rate. This would have implications for how we understand regulation of both stem and non-stem cell populations in many other tissues and organs. 
A
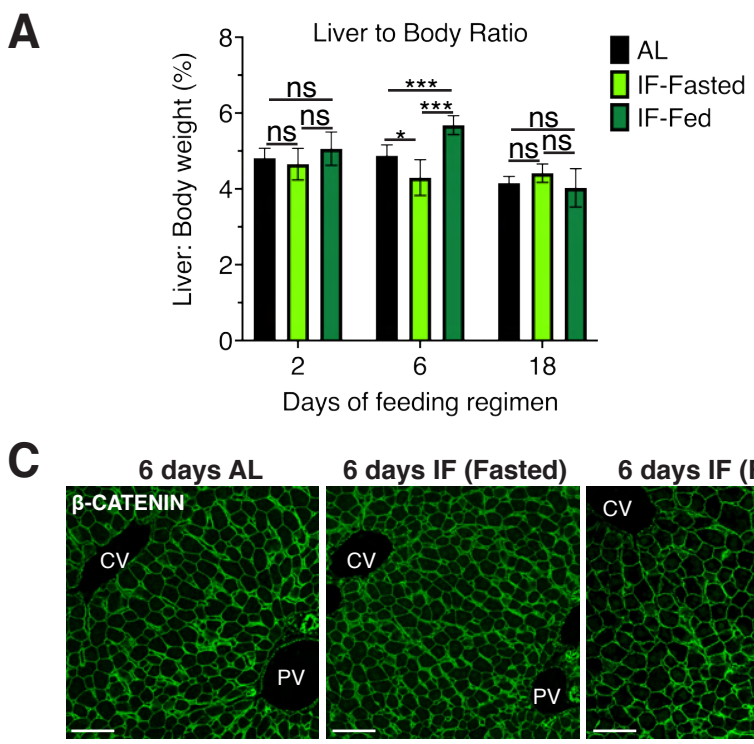

E

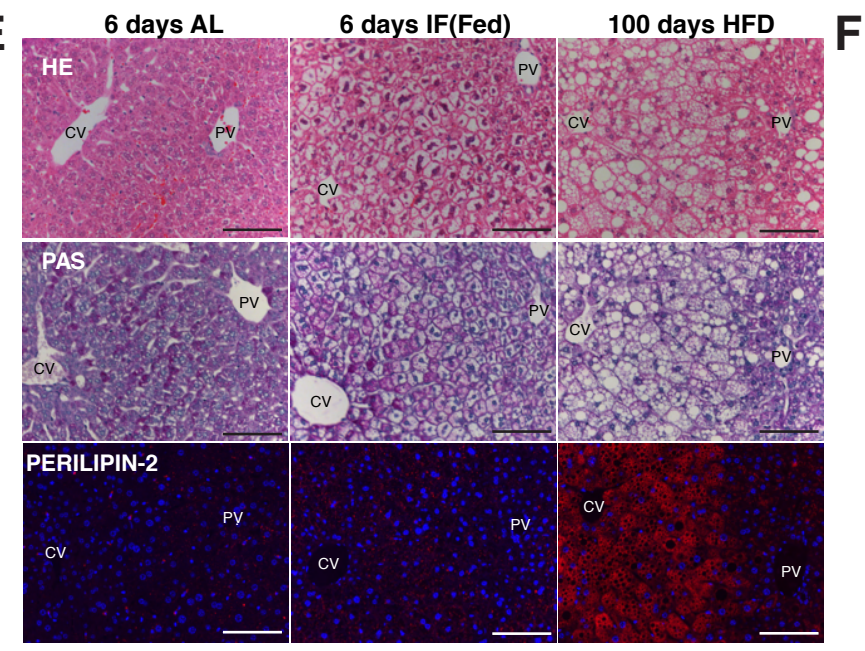

B
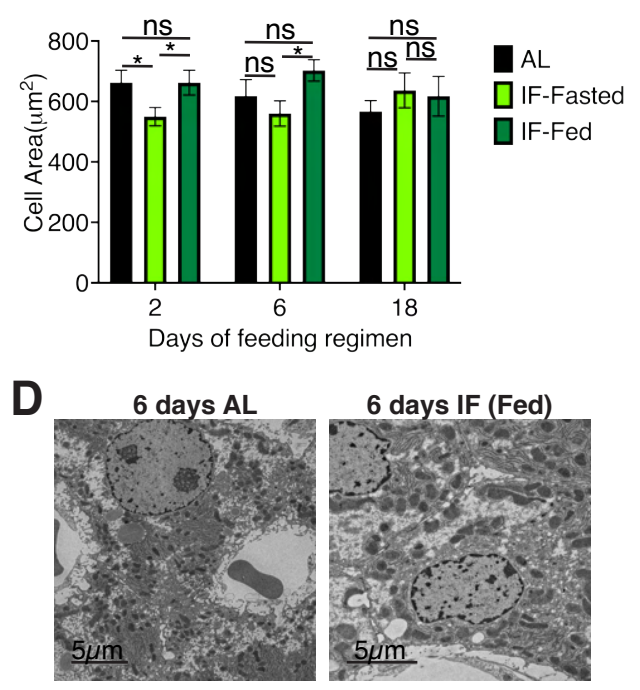

$\mathbf{F}$

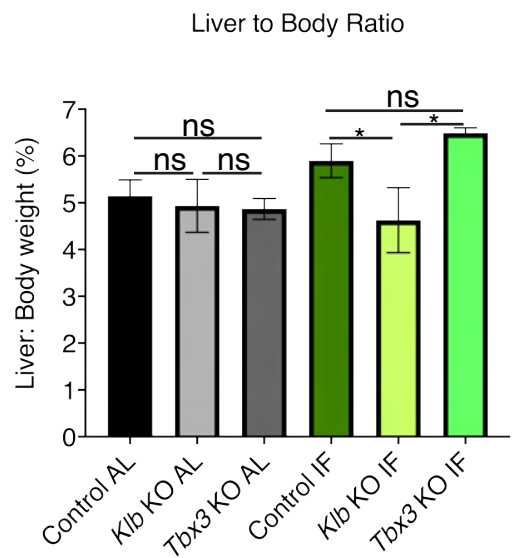

Figure. 4. Intermittent fasting disrupts the hepatostat. (A) Ratio of liver weight to body weight significantly changes between fasted and fed states during early time-frames of IF. 2-way Anova. N=7. (B) Hepatocyte cell area changes between fasted and fed states during IF. 2-way Anova. N=4. (C) Immunofluorescence images for cell membrane marker ß-CATENIN highlighting increase in cell size during IF. (D) Electron microscopy of AL and IF livers shows changes in ribosome and mitochondria content. (E) Hepatocyte swelling occurs in IF livers compared to AL, however is not related to buildup of glycogen (PAS) or lipid droplets (PERILIPIN2). HFD, high fat diet control for PERILIPIN2. (F) Ratio of liver weight to body weight in Klb KO, Tbx3 KO and Control animals after 1 week of AL or IF-treatment. 1-way Anova. ${ }^{* * *} \mathrm{P}<0.001,{ }^{*} \mathrm{P}<0.05$, ns., not significant. Error bars indicate standard deviation. Scale bar, $100 \mu \mathrm{m}$ unless otherwise stated.

\section{Methods}

Mouse strains, husbandry, and experimental methods

All experiments were done on adult, 8-12-week-old, male mice, unless otherwise noted. Wild type C57BL/6J mice, R26-CreERT2 (15), R26-Confetti (16), Axin2-rtTA (17), TetO-H2B-GFP (18) and $R 26-\operatorname{Cas} 9$ (31) strains were obtained from The Jackson Laboratory (JAX). Tbx3 flox 
mice were a gift from Dr. Anne Moon (32). Klb flox mice were a gift from Dr. David Mangelsdorf (33). All mice were housed in the animal facility of Stanford University on a 12-h light/dark cycle $(0700 \mathrm{~h} / 1900 \mathrm{~h})$ ad libitum access to water and food (standard chow diet with $18 \%$ calories derived from fat; $24 \%$ calories from protein and $58 \%$ calories from carbohydrates, Tekland 2918).

For all intermittent fasting, mice were randomly assigned into ad libitum or intermittent fasting groups. Intermittent fasting was performed with total food deprivation and ad libitum access to water from approximately $1900 \mathrm{~h}$ to $1900 \mathrm{~h}$ the following day to implement alternate periods of 24 hour fasting and feeding. Unless specified otherwise in text and figures, all samples were collected for both ad libitum and intermittent fasting groups at $1200 \mathrm{~h}$ to access samples during a neutral metabolic and circadian rhythm time point.

For random cell lineage tracing studies, R26-CreERT2/R26-Confetti mice received intraperitoneal injections of tamoxifen (Sigma, $4 \mathrm{mg} / 25$ grams mouse weight) dissolved in 10\% ethanol/corn oil (sigma) twice with 48 hours between injections. 2 weeks after the last tamoxifen injection, livers where immediately analyzed (T0) or analyzed after an additional 1-3 weeks of ad libitum feeding or intermittent fasting.

For Axin2+ cell lineage tracing studies, Axin2-rtTA; TetO-H2B-GFP mice received doxycycline hyclate (Dox, sigma, $1 \mathrm{mg} / \mathrm{ml}$ ) in drinking water for 5 days. Dox water was then replaced with normal drinking water for 3 days before livers were immediately analyzed(T0) or analyzed after an additional 1 week of ad libitum feeding or intermittent fasting.

For all AAV studies, mice were intraperitoneally injected with $1 \times 10^{11}$ genome copies per mouse at 6-8 weeks of age to induce liver specific depletion of Klb or Tbx3(AAV8-TTR-Cre, Vector Bio Labs, 7102), Fgf15 overexpression (AAV8-TTR-FGF15) or Apc gene editing (AAV8-U6-sgAPC). For AAV studies, an AAV8-Nul $l$ vector containing no transgene (Vector Bio Labs, 7007) was used as a control for AAV infection. For studies that combined AAV injection and Axin2+ cell lineage tracing, mice were first injected with AAV, allowed 3 days to recover and subsequently treated with dox water to induce tracing. All animal experiments and methods were approved by the Institutional Animal Care and Use committee at Stanford University. In conducting research using animals, the investigators adhered to the laws of the United States and regulations of the Department of Agriculture.

\section{Tissue collection, processing, staining, and imaging}

For clonal analysis and KLOTHO immunofluorescence, mice were perfused with 4\% paraformaldehyde (PFA), livers were isolated and further fixed in $4 \%$ PFA for 2 hours at $4{ }^{\circ} \mathrm{C}$. PFA-fixed tissues were washed in PBS and sectioned into 50-200 $\mu \mathrm{m}$ sections using a Compresstome vibrating microtome tissue slicer (VF-310-0Z, Precisionary). Vibratome sections from the median lobe where then permeabilized, stained and cleared using a method developed by the Zerial lab (www.zeriallab.org). The following antibodies were used: GFP (chicken, 1:500, Abcam ab13970), RFP (rabbit, 1:500, Rockland 600-401-379), HNF4-ALPHA (mouse, 1:500; Abcam ab41898) and KLOTHO (rat, 1:200, DSBH KL-115). Vibratome sections were imaged using an SP8 White Light Laser Confocal microscope (Lecia) and a BZ-X800 microscope (Keyence). Confocal image stacks were acquired at 20x magnification and up to $100 \mu \mathrm{m}$ with a step size of $1 \mu \mathrm{m}$ along the Z-axis and processed and analyzed with Imaris software.

For histology and immunohistochemistry, liver was fixed overnight in $10 \%$ formalin at room temperature, dehydrated, cleared in HistoClear (Natural Diagnostics), and embedded in 
paraffin. Sections were cut at $5 \mu \mathrm{m}$ thickness, de-paraffinized, re-hydrated and processed for further staining via immunofluorescence or in situ hybridization assays as described below.

For histology, formalin-fixed paraffin-embedded liver sections were sent to the Department of Comparative Medicine's Animal Histology Services for H\&E and PAS staining. For sirus red staining, slides were deparaffinated in HistoClear (Natural Diagnostics), hydrated in graded ethanol series and rinsed in distilled water. Slides were covered in $0.1 \%$ Sirius Red in saturated picric acid F-357-2 (Rowley Biochemical) for 1 hour and washed in two changes of acidified water $(0.5 \%$ glacial acetic acid). They were then dehydrated in ethanol, cleared in HistoClear and mounted in resinous medium.

For immunofluorescence, sections of formalin-fixed paraffin-embedded livers were subjected to antigen retrieval with Tris buffer $\mathrm{PH}=8.0$ (Vector Labs H-3301) in a pressure cooker. They were then blocked in 5\% normal donkey serum in PBS containing $0.1 \%$ Triton-X, in combination with the Avidin/Biotin Blocking reagent (Vector Labs SP-2001). Sections were incubated with primary and secondary antibodies and mounted in Prolong Gold with DAPI medium (Invitrogen). Biotinylated goat antibody (Jackson Immunoresearch 705-065-147) was applied to section stained with TBX3, before detection with Streptavidin-647. GS and BETACATENIN staining was performed with the Mouse-on-Mouse detection kit (Vector Labs) according to manufacturer's protocol. The following antibodies were used: GFP (chicken, 1:500, Abcam ab13970), TBX3(goat, 1:50, Santa Cruz sc-17871), GS (mouse, 1:500; Millipore MAB302), BETA-CATENIN (mouse, 1:100, BD Transductions laboratory 610154), HNF4ALPHA (mouse, 1:50; Abcam ab41898) and PERILIPIN 2 (1:100, Progen Biotechnik GP46). Samples were imaged at 20X magnification using an Sp8 Confocal or a Zeiss Imager Z.2 and processed and analyzed with ImageJ software.

\section{RNAscope in situ hybridization}

In situs were performed using the RNAscope 2.5 HD Duplex Reagent Kit (Advanced Cell Diagnostics) according to the manufacturer's instructions. Images were taken at 20x magnification on a Zeiss Imager Z.2 and processed using ImageJ software. Probes used in this study were Cyp2f2 (target region: 555-1693) and Cyp2el (target region: 458-1530).

\section{Fibrosis Assay}

Bright-field images were collected on a Zeiss Imager Z.2. Fibrosis levels were assessed qualitatively and graded as: absence of fibrosis $=$ None, some fibrosis $=1$, relatively abundant fibrosis $=2$.

\section{Clone size, number, and location}

To quantify clone size, threshold of fluorescent channels was lowered so that clear cell and nuclear boundaries could be distinguished. 1 large-stitched image with an area of $1.8 \times 1.8 \times 0.1$ $\mathrm{mM}^{3}$ mouse was taken from 2 representative vibratome sections from each mouse at each time point. Only clones completely within the tissue sample were analyzed. We counted the total number of clones from the 6 representative images. Because images were of equal area, clone numbers can be compared to each other between time points. Clones containing a cell located within 3 cell distances from the portal vein or bile duct were classified as periportal; clones 
containing a cell within 3 cell distances from the central vein were classified as pericentral; clones not meeting either criterion were classified as midlobular.

\section{Hepatocyte nuclei isolation and analysis}

For hepatocyte nuclei isolation, liver lobes from mice were homogenized in cold 1\% formaldehyde in PBS with a loose pestle and Dounce homogenizer. Samples where then fixed for $10 \mathrm{~min}$ at room temperature followed by incubation for $5 \mathrm{~min}$ with glycine at a final concentration of $0.125 \mathrm{M}$. Samples were centrifuged at $300 \mathrm{~g}$ for $10 \mathrm{~min}$, at $4^{\circ} \mathrm{C}$. Pellets were washed in PBS and re-suspended with $10 \mathrm{ml}$ cell lysis buffer $(10 \mathrm{mM}$ Tris-HCL, $10 \mathrm{mM} \mathrm{NaCl}$, $0.5 \%$ IGEPAL) and filtered through $100 \mu \mathrm{m}$ cell strainers. A second round of homogenization was performed by 15-20 strokes with a tight pestle. Nuclei were pelleted at $2000 \mathrm{~g}$ for $10 \mathrm{~min}$ at $4^{\circ} \mathrm{C}$ and re-suspended in $0.5 \mathrm{ml} \mathrm{PBS}$ and $4.5 \mathrm{ml}$ of pre-chilled $70 \%$ ethanol and stored at $-20^{\circ} \mathrm{C}$ before downstream GFP content and ploidy analysis by flow cytometry.

Right before flow cytometry, 1 million nuclei were resuspended in PBS and stained with FxCycle PI/RNase (Thermo Fisher, F10797) staining solution for 15-30 minutes at room temperature. Cells were analyzed on a FACS ARIA II (BD). Data were processed with FACS Diva 8.0 software (BD) and FlowJo v10 (FlowJo). Doublets were excluded by FSC-W $\times$ FSC-H and SSC-W $\times \mathrm{SSC}-\mathrm{H}$ analysis. Single-stained channels were used for compensation and fluorophore minus one control was used for gating.

\section{Real-time PCR measurement}

Liver samples were homogenized in TRIzol (Invitrogen) with a bead homogenizer (Sigma). Total RNA was purified using the RNeasy Mini Isolation Kit (Qiagen) and reverse-transcribed (High Capacity cDNA Reverse Transcription Kit, Life Technologies) according to the manufacturer's protocol. Quantitative RT-PCR were performed with TaqMan Gene Expression Assays (Applied Biosystems) on a StepOnePlus Real-Time PCR System (Applied Biosystems). Relative target gene expression levels were calculated using the delta-delta CT method (34). Gene Expression Assays used were Gapdh (Mm99999915_g1) as control, Klb (Mm00473122_m1), Axin2 (Mm00443610_m1), Fgf15(Mm00433278_m1), Tbx3 (Mm01195719_m1), E2f7 (Mm00618098_m1), and E2f8 (Mm01204165_g1), Mki67 (Mm01278617_m1), Ccnd1 (Mm00432359_m1).

\section{Single Cell RNA Sequencing}

Hepatocytes were isolated from livers of 8-week-old C57BL/6J mice that had been intermittent fasted for 1 week or ad libitum fed using a two-step collagenase perfusion technique as previously described (35). Collections were performed at 1000h and during the feeding cycle of IF. For each feeding regimen, 3 livers were collected and processed as 3 individual samples. For each sample, 2,000 hepatocytes were loaded to target $\sim 1000$ cells after recovery according to the manufacturer's protocol. Single cell libraries were prepared using the 10x Genomics Chromium Single Cell 3" Reagents Kit V3. Single cell libraries were loaded on an Illumina NovaSeq 6000 instrument with NovaSeq S2 v.1.5 Reagent Kits with the following reads: 28 bases Read 1 (cell barcode and unique molecular identifier (UMI)), 8 bases i7 Index 1 (sample index), 91 bases Read 2 (transcript). 
Sample demultiplexing, barcode processing, single-cell counting and reference genome mapping were performed using the Cell Ranger Software (v3.1.0, mm10 ref genome) accordingly to the manual. All samples were normalized to present the same effective sequencing depth by using Cell Ranger aggr function. The dimensionality reduction by principal components analysis (PCA), the graph-based clustering and UMAP visualization were performed using Seurat (v3.0, R package). Genes that were detected in less than three cells were filtered out, and cells were filtered out with greater than 10 percent of mitochondrial genes and with fewer than 200 or greater than 50000 detected genes.

For cell clustering, R software was used to sort cells into either pericentral (PC), midlobular (Mid), and periportal (PP) classes based on the greatest expression of biomarkers Cyp2e1, Cypla2, Glul (for PC), Hamp and Igfbp2 (for Mid), Cyp2f2 and Cps1 (for PP).

\section{Generation of $A A V$ Expression Vectors}

The AAV-TTR-FGF15 virus was produced from the complementary stand AAV construct, csAAV-TTR-CRE plasmid (kind gift of Holger Willenbring). The CRE gene was excised by digestion with SalI (NEB). The Fgf15 gene (GenBank: BC021328; cloneID: 5066286) was amplified for assembly into the SalI cut AAV-TTR backbone with NEB HiFi Builder using the primers: (FWD) 5'ggagaagcccagctgGTCGACGCCACCATGGCGAGAAAGTGGAACGG 3' and (REV) 5' atcagcgagctctaGTCGACTCATTTCTGGAAGCTGGGACTCTTCAC 3'. The two fragments were assembled with NEB HiFi builder and cloned in NEB Stable e. coli.

The AAV-sgApc virus was produced from the pAAV-Guide-it-Down construct (Clontech Laboratories Inc., 041315) using assembly primers:

(FWD) 5'CCGGAGGCTGCATGAGAGCACTTG3' and

(Rev) 5'AAACCAAGTGCTCTCATGCAGCCT3'3.

AAV-sgApc contains a U6 promoter and an sgRNA targeting the sequence

5'AGGCTGCATGAGAGCACTTG3' in exon 13 of $A p c$.

\section{Acknowledgments}

We thank K. M. Loh for constructive feedback on the manuscript; M. Morri, L. Penland and the Chan Zuckerberg Biohub Community Access Program for use of 10x Genomics equipment and sequencing; Stanford Neuroscience Gene and Vector Virus Core for virus production; and H. Willenbring for sharing the csAAV-TTR-CRE plasmid. Funding: This study was supported by the Howard Hughes Medical Institute (HHMI) and the Stinehart Reed Foundation. A.S. was supported by the Office of the Assistant Secretary of Defense for Health Affairs, through the Peer Reviewed Cancer Research Program, under Award No. W81XWH-17-1-0245. Opinions, interpretations, conclusions, and recommendations are those of the author and are not necessarily endorsed by the Department of Defense. P.W. was supported by the Damon Runyon Cancer Research Foundation (DRSG-28P-19). The Genetics Bioinformatics Service Center of Stanford Cancer Institute's Share Resource Facility was supported by NIH grant P30 CA124435. 


\section{References}

Brandhorst S, Choi IY, Wei M, et al. (2015) A Periodic Diet that Mimics Fasting Promotes Multi-System Regeneration, Enhanced Cognitive Performance, and Healthspan. Cell Metab 22(1):86-99 doi:10.1016/j.cmet.2015.05.012

Cerletti M, Jang YC, Finley LW, Haigis MC, Wagers AJ (2012) Short-term calorie restriction enhances skeletal muscle stem cell function. Cell Stem Cell 10(5):515-9 doi:10.1016/j.stem.2012.04.002

Chen F, Jimenez RJ, Sharma K, et al. (2020) Broad Distribution of Hepatocyte Proliferation in Liver Homeostasis and Regeneration. Cell Stem Cell 26(1):27-33 e4 doi:10.1016/j.stem.2019.11.001

Chen J, Astle CM, Harrison DE (2003) Hematopoietic senescence is postponed and hematopoietic stem cell function is enhanced by dietary restriction. Exp Hematol 31(11):1097-103 doi:10.1016/s0301-472x(03)00238-8

Ertl RP, Chen J, Astle CM, Duffy TM, Harrison DE (2008) Effects of dietary restriction on hematopoietic stem-cell aging are genetically regulated. Blood 111(3):1709-16 doi:10.1182/blood-2007-01-069807

Halpern KB, Shenhav R, Matcovitch-Natan O, et al. (2017) Single-cell spatial reconstruction reveals global division of labour in the mammalian liver. Nature 542(7641):352-356 doi:10.1038/nature21065

He L, Pu W, Liu X, et al. (2021) Proliferation tracing reveals regional hepatocyte generation in liver homeostasis and repair. Science 371(6532) doi:10.1126/science.abc4346

Inagaki T, Choi M, Moschetta A, et al. (2005) Fibroblast growth factor 15 functions as an enterohepatic signal to regulate bile acid homeostasis. Cell Metab 2(4):217-25 doi:10.1016/j.cmet.2005.09.001

Jin Y, Anbarchian T, Wu P, Sarkar A, Fish M, Nusse R (2021) Hepatocyte Cell Cycle Progression Depends on a Transcriptional Repressor Cascade Downstream of Wnt Signaling. bioRxiv:2021.10.15.464616 doi:10.1101/2021.10.15.464616

Lin S, Nascimento EM, Gajera CR, et al. (2018) Distributed hepatocytes expressing telomerase repopulate the liver in homeostasis and injury. Nature 556(7700):244-248 doi:10.1038/s41586-018-0004-7

Matsumoto T, Wakefield L, Tarlow BD, Grompe M (2020) In Vivo Lineage Tracing of Polyploid Hepatocytes Reveals Extensive Proliferation during Liver Regeneration. Cell Stem Cell 26(1):34-47 e3 doi:10.1016/j.stem.2019.11.014

McGrew LL, Hoppler S, Moon RT (1997) Wnt and FGF pathways cooperatively pattern anteroposterior neural ectoderm in Xenopus. Mech Dev 69(1-2):105-14 doi:10.1016/s0925-4773(97)00160-3

Michalopoulos GK (2021) Novel insights into liver homeostasis and regeneration. Nat Rev Gastroenterol Hepatol doi:10.1038/s41575-021-00454-0

Michalopoulos GK, Bhushan B (2021) Liver regeneration: biological and pathological mechanisms and implications. Nat Rev Gastroenterol Hepatol 18(1):40-55 doi:10.1038/s41575-020-0342-4

Mihaylova MM, Sabatini DM, Yilmaz OH (2014) Dietary and metabolic control of stem cell function in physiology and cancer. Cell Stem Cell 14(3):292-305

doi:10.1016/j.stem.2014.02.008 
Miyaoka Y, Ebato K, Kato H, Arakawa S, Shimizu S, Miyajima A (2012) Hypertrophy and unconventional cell division of hepatocytes underlie liver regeneration. Curr Biol 22(13):1166-75 doi:10.1016/j.cub.2012.05.016

O'Brien LE, Soliman SS, Li X, Bilder D (2011) Altered modes of stem cell division drive adaptive intestinal growth. Cell 147(3):603-14 doi:10.1016/j.cell.2011.08.048

Perugorria MJ, Olaizola P, Labiano I, et al. (2019) Wnt-beta-catenin signalling in liver development, health and disease. Nat Rev Gastroenterol Hepatol 16(2):121-136 doi:10.1038/s41575-018-0075-9

Potthoff MJ, Boney-Montoya J, Choi M, et al. (2011) FGF15/19 regulates hepatic glucose metabolism by inhibiting the CREB-PGC-1alpha pathway. Cell Metab 13(6):729-38 doi:10.1016/j.cmet.2011.03.019

Potthoff MJ, Kliewer SA, Mangelsdorf DJ (2012) Endocrine fibroblast growth factors 15/19 and 21: from feast to famine. Genes Dev 26(4):312-24 doi:10.1101/gad.184788.111

Snippert HJ, van der Flier LG, Sato T, et al. (2010) Intestinal crypt homeostasis results from neutral competition between symmetrically dividing Lgr5 stem cells. Cell 143(1):134-44 doi:10.1016/j.cell.2010.09.016

Suzuki A, Sekiya S, Buscher D, Izpisua Belmonte JC, Taniguchi H (2008) Tbx3 controls the fate of hepatic progenitor cells in liver development by suppressing p19ARF expression. Development 135(9):1589-95 doi:10.1242/dev.016634

ten Berge D, Brugmann SA, Helms JA, Nusse R (2008) Wnt and FGF signals interact to coordinate growth with cell fate specification during limb development. Development 135(19):3247-57 doi:10.1242/dev.023176

Tumbar T, Guasch G, Greco V, et al. (2004) Defining the epithelial stem cell niche in skin. Science 303(5656):359-63 doi:10.1126/science.1092436

Uriarte I, Fernandez-Barrena MG, Monte MJ, et al. (2013) Identification of fibroblast growth factor 15 as a novel mediator of liver regeneration and its application in the prevention of post-resection liver failure in mice. Gut 62(6):899-910 doi:10.1136/gutjnl-2012-302945

Ventura A, Kirsch DG, McLaughlin ME, et al. (2007) Restoration of p53 function leads to tumour regression in vivo. Nature 445(7128):661-5 doi:10.1038/nature05541

Wang B, Zhao L, Fish M, Logan CY, Nusse R (2015) Self-renewing diploid Axin2(+) cells fuel homeostatic renewal of the liver. Nature 524(7564):180-5 doi:10.1038/nature14863

Wei Y, Wang YG, Jia Y, et al. (2021) Liver homeostasis is maintained by midlobular zone 2 hepatocytes. Science 371(6532) doi:10.1126/science.abb1625

Yilmaz OH, Katajisto P, Lamming DW, et al. (2012) mTORC1 in the Paneth cell niche couples intestinal stem-cell function to calorie intake. Nature 486(7404):490-5 doi:10.1038/nature11163

Yu HM, Liu B, Costantini F, Hsu W (2007) Impaired neural development caused by inducible expression of Axin in transgenic mice. Mech Dev 124(2):146-56 doi:10.1016/j.mod.2006.10.002 


\section{Supplementary Figures}

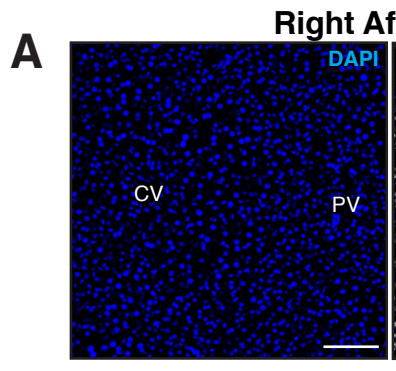

B
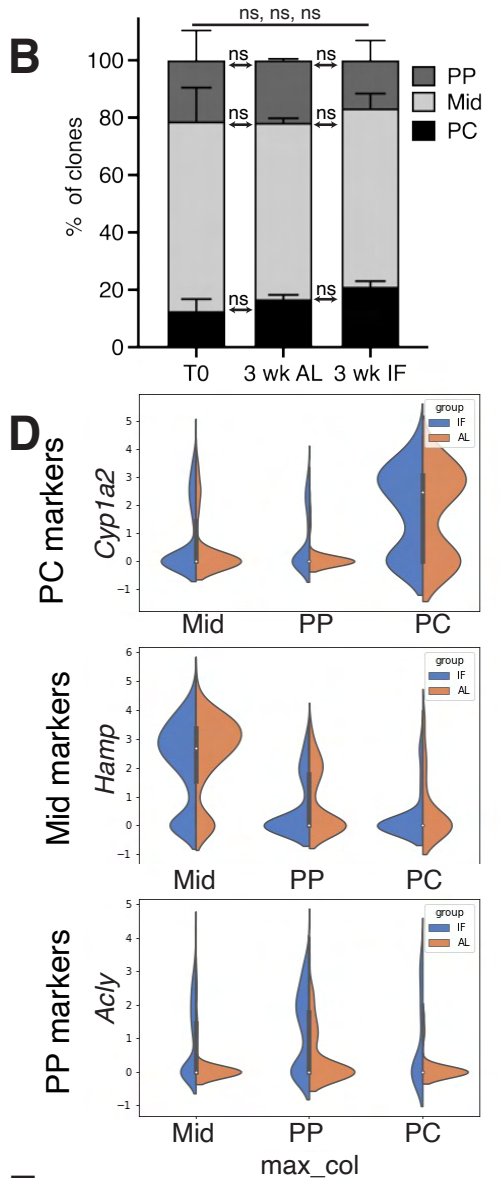

$\mathbf{F}$

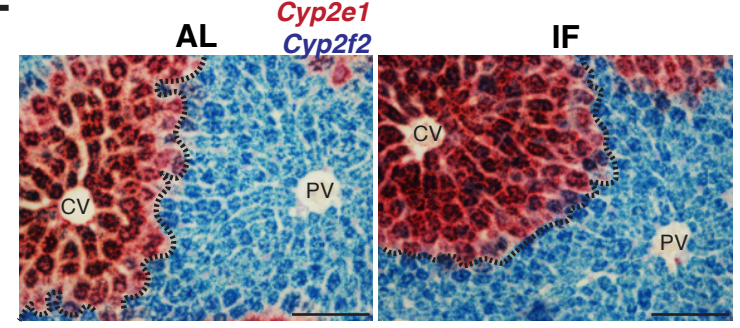

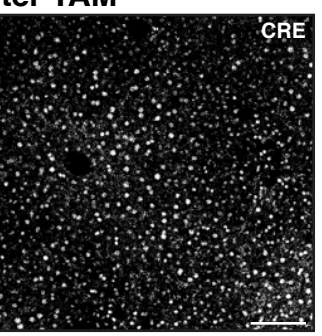

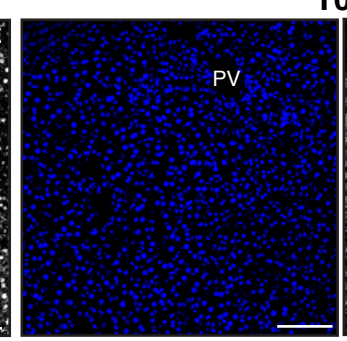

To

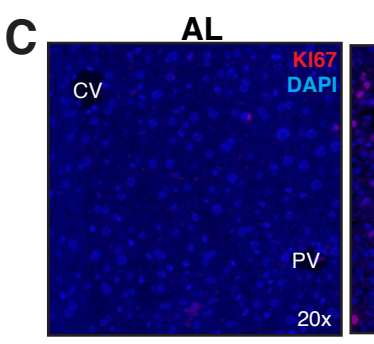

IF (re-fed)
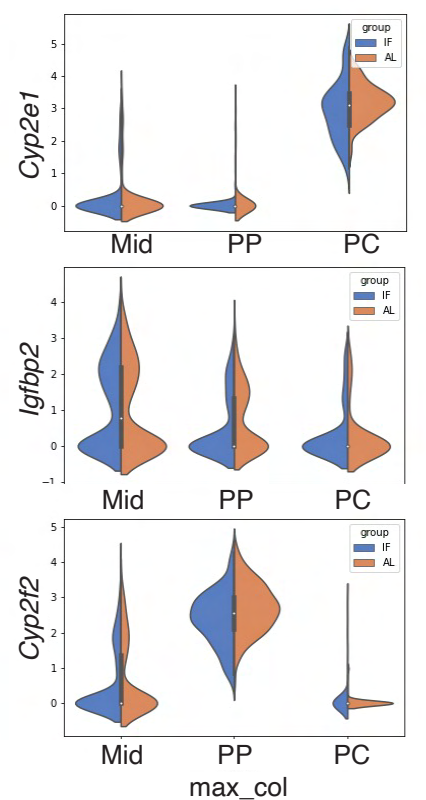

G

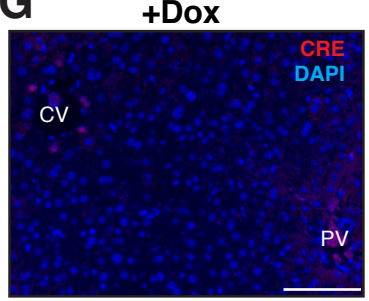

E
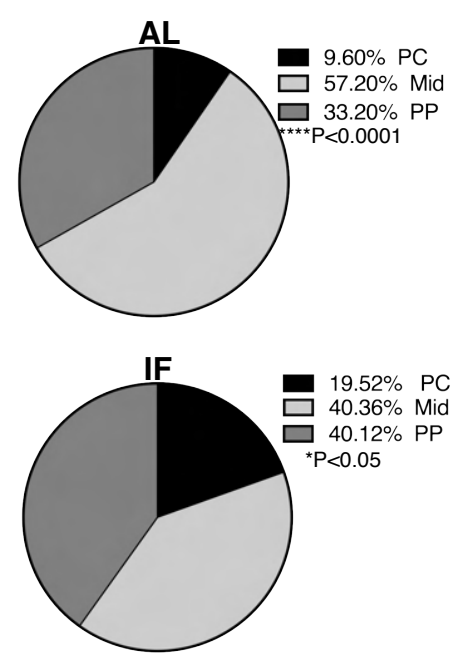

TO

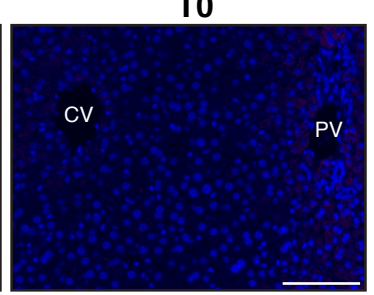

Figure S1 (related to Figure 1). Hepatocyte dynamics in ad libitum fed and intermittent fasted animals. (A) Tamoxifen clearance before AL and IF treatment was confirmed by loss of CRE immunofluorescence at T0 in livers from R26-CreER; R26-confetti mice. (B) Percent of clones in different locations in the liver lobule at T0, 3 wk AL and 3 wk IF in R26-CreER; R26-confetti mice. (C) Immunofluorescence for Ki67 in 1 week AL and1 week IF livers. (D) Violin plots of zonal marker gene expression in single hepatocytes from AL and IF livers classified as PC, Mid and PP hepatocytes. (E) Pie chart showing percentages of hepatocytes with PC, Mid and PP transcript expression in 
bioRxiv preprint doi: https://doi.org/10.1101/2021.10.16.464650; this version posted October 16, 2021. The copyright holder for this preprint (which was not certified by peer review) is the author/funder. All rights reserved. No reuse allowed without permission.

AL and IF livers. 1way Anova. N=3. (F) RNAscope of IF and AL livers using Cyp2e1 (PC marker) and Cyp2f2 (PP marker) probes.(G) Doxycycline clearance was confirmed by loss of CRE immunofluorescent at $\mathrm{T} 0$ in livers from Axin2rtTA; H2BGFP mice. $* * * \mathrm{P}<0.001,{ }^{*} \mathrm{P}<0.05$, ns., not significant. Error bars indicate standard deviation. Scale bar, $100 \mu \mathrm{m}$. 